\title{
Aggregated Energy Storage for Power System Frequency Control: A Finite-Time Consensus Approach
}

\author{
Yu Wang, Member, IEEE, Yan Xu, Member, IEEE, Yi Tang, Member, IEEE, and Kai Liao, Member, \\ IEEE, Mazheruddin H. Syed, Student Member, IEEE, Efren Guillo-Sansano, Student Member, IEEE \\ and Graeme Burt, Member, IEEE
}

\begin{abstract}
In future power systems, widespread small-scale energy storage systems (ESSs) can be aggregated to provide ancillary services. In this context, this paper aims to integrate energy storage aggregators (ESAs) into the load frequency control (LFC) framework for power system frequency control. Firstly, a system disturbance observer is designed to supplement the secondary frequency control, where the ESA can respond to the estimated disturbance and accelerate the system frequency recovery. Then, within the ESA, a finite-time leader-follower consensus algorithm is proposed to control the small-scale ESSs via sparse communication network. This algorithm ensures that the ESAs can track the frequency control signals and the state-ofcharge balancing among each ESS in finite-time. The external characteristics of the ESA will resemble to that of one large-scale ESS. Numerical examples demonstrate the convergence of the ESA under different communication graphs. The effectiveness of the entire framework for power system frequency control is validated under a variety of scenarios.
\end{abstract}

Index Terms - Frequency control, consensus algorithm, energy storage systems, aggregator, demand response.

\section{INTRODUCTION}

$\mathrm{I}_{\mathrm{p}}^{\mathrm{N}}$ $\mathrm{N}$ recent years, renewable energy sources (RESs), such as photovoltaic (PV) panels and wind turbines, have been widely integrated into power grids [1]. In Singapore, the PV penetration level is expected to increase to about $10 \%$ in less than a decade from now on [2]. Although RESs are environmentally friendly, their high variability and uncertainty have introduced significant challenges to power system's operation and control [3]. Conventionally, the power imbalance between generation and load consumption is addressed by synchronous generators in large power plants. As the increasing penetration level of RESs and subsequent replacement of synchronous generators, not only the system inertia but also the system frequency reserves will be reduced. When a system disturbance occurs, a power system with less inertia and frequency regulation reserves tends to experience more severe frequency deviations [4], [5]. Therefore,

This work is supported by Energy Innovation Research Programme Energy Storage Grant (NRF2015EWT-EIRP002-007), and also supported in part by the European Liaison on Electricity Committed Towards longterm Research Activity (ELECTRA) Integrated Research Program (IRP).

Y. Wang, Y. Xu, Y. Tang, and K. Liao are with School of Electrical and Electronic Engineering, Nanyang Technological University, Singapore.

M. Syed, E.Guillo-Sansano, and G. Burt are with Department of electronic and Electrical Engineering, University of Strathclyde, Glasgow, UK. additional frequency regulation resources and corresponding control methods are needed for high RES penetrated power systems.

Energy storage systems (ESSs) have drawn significant attention and have been progressively deployed in modern power systems, due to their high flexibility to contribute to power system operation and control [6]. The ESSs can be classified according to their capacity and ownership. The large-scale centralized ESSs which are usually owned by the utility grid can have the capacity up to tens of MWh. For example, the Energy Market Authority of Singapore has raised the target to install 4.4 MWh island-wide ESSs by the year of 2020 [7]. The large-scale grid ESSs have been studied in the literature to mitigate $\mathrm{PV} /$ wind fluctuations [8] and power system frequency regulation [9].

Except for large-scale ESSs, there are also a large amount of small-scale ESSs widespread in the power system. The small-scale distributed ESSs such as plug-in electric vehicles (PEV) and community ESSs usually have the capacity up to hundreds of kWh [6]. These small-scale distributed ESSs also have large potential in future frequency ancillary services market. In the literature, the mobile ESSs provided by PEVs are used for system frequency regulation through vehicle-togrid or grid-to-vehicle operations [10]. The stationary ESSs owned by both the utility or customers can participate in system frequency and voltage regulation through controlled charging and discharging [11], [12]. However, the distributed ESSs located at the demand side are usually larger in numbers but smaller in capacity. Therefore, their contributions to the power system are hard to account individually. The concept of aggregator offers the feasibility for large number of smallscale ESSs to participate in ancillary services (such as frequency regulation and restoration) to gain additional income [13], [14]. In an aggregator, the geographically dispersed units can cooperate to achieve a common objective through communication networks. Correspondingly, the energy storage aggregator (ESA) will have much larger power and capacity rating compared to a single ESS and it can be viewed as one entity for the system operator. One major objective of this paper is to study the formation and dynamic response of these ESAs.

In terms of control methods, centralized control structures of ESSs have been proposed in some literatures for power system frequency control. In [15], a fuzzy-logic based control 
for wind farms and ESSs within the load frequency control (LFC) scheme has been proposed. In [16], a master-slave based centralized control of distributed ESS has been proposed for mitigating voltage and frequency deviations. Similarly, a centralized coordinated control for EVs and ESSs participating in LFC has been presented in [17]. The major concern of the centralized control structure is the requirement of a central communication infrastructure. This bottleneck limits the support for plug-and-play functions which is highly desired for widespread distributed devices.

As an alternative method, distributed control has raised considerable attention in the power system community. Unlike the centralized control, this control scheme does not need full communication access as each controllable device only communicates with its neighbours, which is more suitable for widespread distributed resources in the distribution network. The idea of consensus based distributed control is to achieve fair utilization of available devices by exchanging information through sparse communication networks. This control algorithm has firstly been applied to distributed control for a group of PV systems and ESSs for distribution networks voltage control in [18], [19]. In [20], the leader-follower consensus control of responsive load aggregators has been proposed for LFC. This research work has been further extended in [21], where a compound control strategy including model predictive control and consensus-based distributed control has been designed to control ESSs in LFC scheme. The state-of-charge (SoC), as the key performance indicator of ESSs is not considered in [19], [21]. Besides, the convergence of the above method in finite-time is not guaranteed. As a result, the convergence time may be too long, and the frequency performance cannot be satisfactory. By contrast, the finite-time consensus can ensure the convergence of participants in finite time, which can effectively enhance the overall system dynamic performance. In [22], a finite-time distributed cooperative voltage unbalance compensation method for microgrid is proposed. In [23], a finite-time distributed voltage and frequency restoration strategy is proposed for islanded AC microgrids. Yet so far, little research has been reported using the finite-time consensus approach to control both the power outputs and SoCs for an aggregator of ESSs simultaneously.

Compared with previous research, the contribution of this paper is twofold: 1) a novel frequency control framework is proposed to integrate the ESA into the power system LFC. A system disturbance observer is proposed to supplement the secondary frequency control of ESA. The high-frequency system disturbance in one control area can be extracted and compensated by the ESA. Compared with the conventional proportional-integral (PI) based secondary frequency control methods, the proposed control scheme exploits the full potential of fast response speed of ESAs, thereby improving system frequency recovery speed; 2) a leader-follower finitetime consensus algorithm is proposed to control ESSs in the ESA. This algorithm ensures power tracking and SoC balancing of ESSs in finite-time, allowing for desired dynamic performance to be obtained through a proper design. For the proposed consensus-based ESA, first, a virtual leader will update the reference power and energy states based on the frequency control signal. Then the reference states are received by the pinning ESSs and transmitted to all ESSs through predefined communication networks. The power and energy states of each ESS will reach consensus at steady-state. Compared with the existing method, the benefits of the proposed ESA with finite-time power tracking and SoC balancing are: (i) it avoids unintentional switching off of certain ESSs when they reach their SoC limits, (ii) the ESA can maintain a larger power rating for a longer time, (iii) it is convenient for the upper level controller to manage the SoCs of the entire aggregator, and (iv) it offers plug-and-play capability, i.e. when a new ESS joins the aggregator for frequency control, its SoC can autonomously be balanced with other ESSs.

\section{Proposed FreQuency ConTROL FrameWork}

\section{A. System Overview}

An overview of the proposed frequency control framework is shown in Fig. 1. As highlighted in Fig. 1, there are two contributions of this paper: (i) a novel frequency control framework that incorporates a system disturbance observer to supplement the secondary frequency control, and (ii) a leaderfollower finite-time consensus algorithm to control ESSs within an ESA. In this section, the multi-area LFC model and the proposed disturbance observer are introduced. The detailed design of the ESA based on finite-time consensus algorithm is presented in Section III.

Under the proposed frequency control framework, the frequency control signal of the ESA is comprised of two parts: (i) The AGC signal from the secondary frequency control with a predefined participation factor $\alpha_{E S A}$. It should be noted that the AGC signal is shared by both generators and ESAs in each control area. (ii) The system disturbance signal estimated by the system disturbance observer. The objective of this design is to accelerate the frequency recovery speed as compared to the conventional PI based secondary frequency control methods. The frequency control signal of the ESA is further shared among each ESS through the proposed finite-time consensus algorithm.

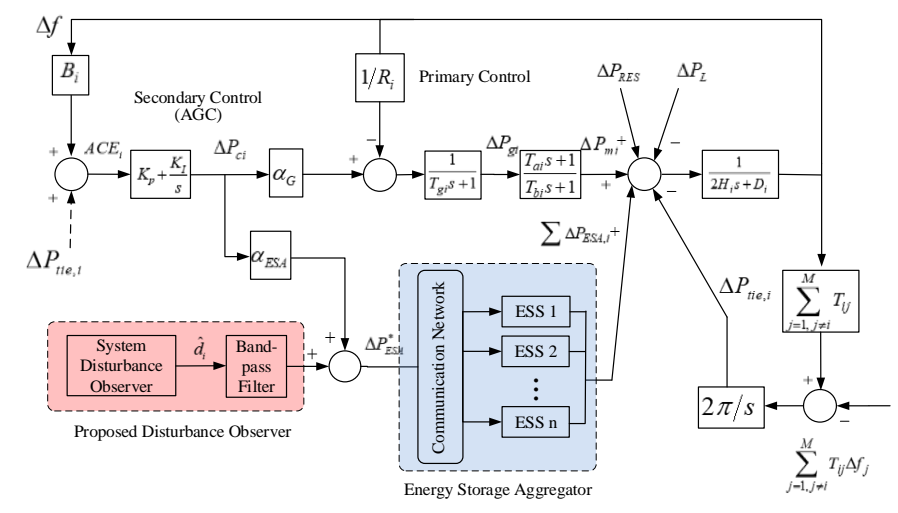

Fig. 1. An overview of the proposed frequency control framework. 


\section{B. Multi-Area LFC Model with ESAs}

Consider an interconnected power system with $M$ control areas indexed by $i=1,2, \ldots M$. The LFC model, including RESs and ESAs, of the $i$ th control area is presented in Fig. 1. The aim of primary control is to contain the frequency deviation caused by power imbalance in any control area, while the secondary control, i.e. automatic generation control (AGC), aims to recover the frequency back to the nominal value [5]. The system dynamics of the $i$ th area can be represented by the following differential equations:

$$
\begin{gathered}
\Delta f_{i}^{\&}(t)=-\frac{D_{i}}{2 H_{i}} \Delta f_{i}(t) \\
+\frac{1}{2 H_{i}}\left(\Delta P_{m i}(t)-\Delta P_{L, i}(t)+\Delta P_{R E S, i}(t)-\Delta P_{t i e, i}(t)+\Delta P_{E S A, i}(t)\right) \\
\Delta \int_{m i}^{\&}(t)=-\frac{1}{T_{b i}} \Delta P_{m i}(t)+\frac{1}{T_{b i}} \Delta P_{g i}(t)+\frac{T_{a i}}{T_{b i}} \Delta P_{g i}^{\&}(t) \\
\Delta P_{g i}^{\&}(t)=-\frac{1}{T_{g i}} \Delta P_{g i}(t)+\frac{1}{T_{g i}} \Delta P_{c i}(t)-\frac{1}{R_{i} T_{g i}} \Delta f_{i}(t) \\
\Delta P_{t i e, i}^{\&}(t)=2 \pi \cdot\left[\sum_{j=1, j \neq i}^{M} T_{i j}\left(\Delta f_{i}(t)-\Delta f_{j}(t)\right)\right]
\end{gathered}
$$

where $\Delta f_{i}$ is the change in system frequency, $\Delta P_{c i}$ denotes control effort of secondary control, $\Delta P_{m i}$ and $\Delta P_{g i}$ are the deviations of generator mechanical output and valve position, respectively. $H_{i}, D_{i}, R_{i}, T_{g i} T_{a i}$ and $T_{b i}$ are the system inertia, load damping coefficient, speed droop, governor and turbine time constants, respectively. $\Delta P_{L, i}, \Delta P_{R E S, i}, \Delta P_{t i e, i}$ and $\Delta P_{E S A, i}$ are power variations of loads, RESs, tie-line and ESAs, respectively, which can be viewed as external disturbances to the system.

The control input for the secondary control is called area control error (ACE) and can be defined as

$$
A C E_{i}(t)=B_{i} \Delta f_{i}(t)+\Delta P_{t i e, i}(t)
$$

where $B_{i}$ the frequency bias factor. In the LFC model, (proportional integral) PI control is commonly utilized for the secondary control, which is to eliminate the ACE for each control area. The PI based AGC controller can be designed as

$$
\Delta P_{c i}(t)=-K_{P} A C E_{i}(t)-K_{I} \int A C E_{i}(t)
$$

where $K_{P}$ and $K_{I}$ are the proportional and integral gains, respectively. In practical condition, the frequency recovery time by secondary frequency control can be as long as $10 \mathrm{~min}$.

Traditionally, synchronous generators in large power plants are responsible for frequency control caused by the load variation in the power system. As the penetration of RESs increase rapidly in power systems, additional disturbances have been added to the original LFC model. Given the high controllability, flexibility, and scalability of ESAs, additional frequency support can be provided by ESAs.

\section{Proposed Disturbance Observer}

The proposed disturbance observer is comprised of a disturbance observer and a band-pass filter in series. The following sub-sections explain the detailed design of the proposed disturbance observer.
Disturbance Observer: The design objective of the disturbance observer is to estimate the real power variations of the control area within which it is deployed, in real-time. Using system dynamical equations (1)-(6) in ith control area, a state-space model $i$ th control area can be obtained as follows:

$$
\boldsymbol{x}_{i}^{\&}(t)=\boldsymbol{A}_{i} \boldsymbol{x}_{i}(t)+\boldsymbol{B}_{i} \mu_{i}(t)+\boldsymbol{F}_{i} \Delta P_{d i}(t)
$$

where the state variable $\boldsymbol{x}_{i}=\left[\begin{array}{ccc}\Delta f_{i} & \Delta P_{m i} & \Delta P_{g i}\end{array}\right]^{T}$, the system input $\mu_{i}=\Delta P_{c i}$, the system disturbance $\Delta P_{d i}=\Delta P_{R E S, i}-\Delta P_{L, i}$ $-\Delta P_{t i e, i}+\Delta P_{E S A, i}$. It is noted that the power output of ESA is also included in the system disturbance. Thus, with proper control of the ESA, the observed system disturbance can be reduced.

$$
\begin{gathered}
\boldsymbol{A}_{i}=\left[\begin{array}{ccc}
-D_{i} / 2 H_{i} & -1 / 2 H_{i} & 0 \\
-T_{a} / R_{i} T_{g} T_{b} & -1 / T_{b i} & \left(1 / T_{b i}-T_{a i} / T_{g i} T_{b i}\right) \\
-1 / R_{i} T_{g i} & 0 & -1 / T_{g i}
\end{array}\right], \boldsymbol{B}_{i}=\left[\begin{array}{c}
0 \\
0 \\
1 / T_{g i}
\end{array}\right], \\
\boldsymbol{F}_{i}=\left[\begin{array}{lll}
-1 / 2 H_{i} & 0 & 0
\end{array}\right]^{T} .
\end{gathered}
$$

With the state space model (7) of the $i$ th area, the system disturbance observer can be designed as [24]:

$$
\left\{\begin{array}{c}
\boldsymbol{x}_{i}^{\&}(t)=\boldsymbol{A}_{i} \hat{\boldsymbol{x}}_{i}(t)+\boldsymbol{B}_{i} \mu_{i}(t)+\boldsymbol{F}_{i} \hat{d}_{i}(t)+\boldsymbol{R}\left(\boldsymbol{y}_{i}(t)-\hat{\boldsymbol{y}}_{i}(t)\right) \\
\hat{\boldsymbol{y}}_{i}(t)=\boldsymbol{C} \hat{\boldsymbol{x}}_{i}(t)
\end{array}\right.
$$

where $\boldsymbol{C}$ is an identity matrix, $\boldsymbol{C}=\operatorname{diag}\{1,1,1\}, \boldsymbol{R}$ is constant gain matrix, $\hat{d}_{i}$ is the estimated system disturbance.

With the estimated states $\hat{\boldsymbol{x}}_{\boldsymbol{i}}$, the system disturbance can be estimated as follows:

$$
\left\{\begin{array}{c}
\hat{d}_{i}(t)=\xi_{i}(t)+\boldsymbol{M} \hat{\boldsymbol{x}}_{i}(t), \\
\xi_{i}(t)=-\boldsymbol{M}\left(\boldsymbol{A}_{i} \hat{\boldsymbol{x}}_{i}(t)+\boldsymbol{B}_{i} \mu_{i}(t)+\boldsymbol{F}_{i} \hat{d}_{i}(t)\right)+d_{i}^{\&}(t)
\end{array}\right.
$$

where $\xi_{i}$ is an auxiliary variable, $\boldsymbol{M}$ is a constant gain matrix.

Defining the state estimation error and disturbance estimation error as $\overline{\boldsymbol{x}}_{i}=\boldsymbol{x}_{i}-\hat{\boldsymbol{x}}_{i}$ and $\bar{d}_{i}=d_{i}-\hat{d}_{i}$, the output estimation error can be obtained with $\boldsymbol{C} \bar{x}_{i}$.

With (8) and (9), the estimated state error dynamics become:

$$
\tilde{\boldsymbol{x}}_{i}^{\mathrm{K}}(t)=\left(\boldsymbol{A}_{i}-\boldsymbol{R} \boldsymbol{C}\right) \overline{\boldsymbol{x}}_{i}(t)+\boldsymbol{F}_{i} \bar{d}_{i}(t)
$$

Combining the disturbance estimation error and state estimation error:

$$
\omega_{i}(t)=\bar{A}_{i} \omega_{i}(t)
$$

where $\boldsymbol{\omega}_{i}=\left[\begin{array}{ll}\overline{\boldsymbol{x}}_{i}^{T} & \bar{d}_{i}\end{array}\right]$ and $\overline{\boldsymbol{A}}_{i}=\left[\begin{array}{cc}\boldsymbol{A}_{i}-\boldsymbol{R} \boldsymbol{C} & \boldsymbol{F}_{i} \\ -\boldsymbol{M R C} & 0\end{array}\right]$.

The eigenvalues of the matrix $\overline{\boldsymbol{A}}_{i}$ can be placed arbitrarily by selecting the gain matrixes $\boldsymbol{R}$ and $\boldsymbol{M}$ as:

$$
\operatorname{det}\left[s \boldsymbol{I}-\overline{\boldsymbol{A}}_{i}\right]=\prod_{m=1}^{4}\left(s+\lambda_{i}\right)
$$


where $\lambda_{i}$ are the desired eigenvalues of the system. Through the eigenvalue palcement in (12), the desired dynamics of system disturbance observer can be designed.

Band-Pass Filter: A band-pass filter is designed to extract the magnitude of system disturbance to be compensated by the ESA within the control area. The function of the band-pass filer is illustrated in Fig. 2. The key idea is to decompose the estimated system disturbance, where the high-frequency components of system disturbance within the passband $\left[f_{\mathrm{L}}, f_{\mathrm{H}}\right]$ will be sent to the ESA. Therefore, the ESAs can respond to sudden system disturbance and improve the system frequency response. The very high-frequency components of system disturbance $\left[f_{\mathrm{H}}, \infty\right]$ can be viewed as system noise, while the low-frequency components within $\left[0, f_{\mathrm{L}}\right]$ can be compensated by conventional generators. Generally, $f_{\mathrm{H}}$ is selected to be larger than the reciprocal of response time of the ESA and $f_{\mathrm{L}}$ is selected to cover the most sensitive frequency band [25].

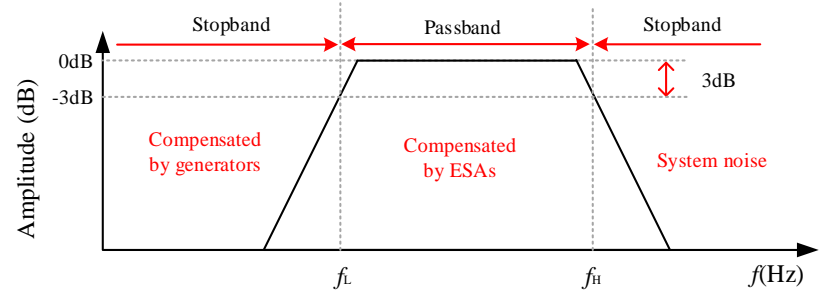

Fig. 2. The function of the band-pass filter.

\section{Finite-Time Consensus-Based ESA}

In a realistic power grid, the small-scale ESSs are usually owned by customers that lack direct communication with the AGC controller. In this condition, the ESSs can be sparsely connected to form an ESA, which can participate in system frequency services as an entity. In this section, detailed design of the ESA based on the finite-time consensus approach and its characteristics will be introduced.

\section{A. Communication Network Preliminaries}

The communication network of an aggregated ESSs (denoted by $i=0,1, \ldots, N$ ) can be described by a graph, which is defined as $G=(\mathcal{V}, \mathcal{E})$ with a set of nodes $\mathcal{V}=\left\{v_{1}, v_{2}, \ldots, v_{N}\right\}$

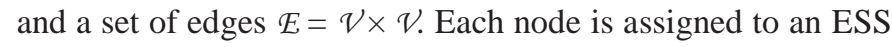
in the ESA, and edges represent communication links for data exchange. If communication links are bidirectional, $\left(v_{i}, v_{j}\right) \in \mathcal{E}$ $\Rightarrow\left(v_{j}, v_{i}\right) \in \mathcal{E} \forall i, j$ the graph is said to be undirected. Otherwise, it is directed. A directed graph is said to have a spanning tree, if there is a root node, such that there is a directed path from the root to any other node in the graph. A matrix called adjacency matrix $\mathcal{A}=\left[a_{i j}\right]$ where $a_{i j}$ can be defined as

$$
a_{i j}= \begin{cases}1, & \text { if }\left(v_{i}, v_{j}\right) \in \mathcal{E} \\ 0, & \text { otherwise. }\end{cases}
$$

The in-degree matrix of $G$ is defined as $\Delta=\operatorname{diag}\left\{\Delta_{i}\right\}$ with $\Delta_{i}=\sum_{j=1}^{N} a_{i j}$. The Laplacian matrix can be represented as

$$
\boldsymbol{L}=\boldsymbol{\Delta}-\mathcal{A} \Rightarrow l_{i j}=\left\{\begin{array}{cc}
-a_{i j}, & i \neq j \\
\sum_{j \in \mathcal{N}_{i}}^{n} a_{i j}, & i=j
\end{array}\right.
$$

The Laplacian matrix $\boldsymbol{L}$ has properties that all row sums of $\mathrm{L}$ are zero.

For the system with a leader (labelled as node 0), the interaction topology can be expressed by directed graph $\bar{G}$, which contains original graph $G$, node $v_{0}$ and edges $\left(v_{i}, v_{0}\right)$ from node $v_{0}$ to other nodes. The leader can send information to followers, but not vice versa. The matrix $\boldsymbol{G}=\operatorname{diag}\left\{g_{1}\right.$, $\left.g_{2}, \ldots g_{N}\right\}$ is used to describe whether each follower directly receive information from the leader, where

$$
g_{i}= \begin{cases}1, & \text { if } \exists\left(v_{i}, v_{0}\right) \\ 0, & \text { otherwise. }\end{cases}
$$

One important property to guarantee that the leaderfollower consensus algorithm with graph $\bar{G}$ will convergence is that the graph $\bar{G}$ contains a spanning tree rooted at node 0 and all the eigenvalues of matrix $\boldsymbol{L}+\boldsymbol{G}$ have positive real parts [26].

\section{B. Finite-Time Consensus Algorithm for ESA}

In this paper, a finite-time leader-follower consensus algorithm is proposed to control each ESS in the ESA. Compared to other consensus algorithms, the finite-time consensus approach guarantees the power tracking and $\mathrm{SoC}$ balancing of each ESS in finite-time. The modelling and design of finite-time consensus based ESA are illustrated as below.

The real power output of $i^{\text {th }}$ ESS is determined by:

$$
P_{E S S, i}(t)=p_{i}(t) \cdot P_{E S S, i}^{\max }
$$

where $P_{E S S, i}^{\max }$ is the power rating (MW) of $i^{\text {th }}$ ESS. $p_{i}$ is the power state of the communication agent associated with $i^{\text {th }}$ ESS. $p_{i} \in[-1,1]$ in practical application considering the power limits of ESS.

The SoC of $i^{\text {th }}$ ESS can be estimated as:

$$
S o C_{i}(t)=S o C_{i}(0)-\int_{t=0}^{T} \frac{\eta_{i} P_{E S S, i}(t)}{3600 \times C_{E S S, i}} d t
$$

where $C_{E S S, i}$ and $S o C_{i}(0)$ are the capacity (MWh) and initial SoC of the $i^{\text {th }}$ ESS. $\eta_{i}$ is the charging or discharing efficiency of the $i^{\text {th }}$ ESS. $C_{E S S, i}$ is multiplied by 3600 to convert the units from hours to seconds.

Define an energy state $e_{i}$ which is equal to the $S o C_{i}$ of $i^{\text {th }}$ ESS. $e_{i} \in[0,1]$ considering the capacity limits of ESS. Combining (17) with (16) and differentiating it with respect to time, the relationship between $p_{i}$ and $e_{i}$ is expressed as:

$$
\underset{t}{\&}(t)=-\frac{\eta_{i} P_{E S S, i}^{\max } p_{i}(t)}{3600 \times C_{E S S, i}}=K_{E S S, i} p_{i}(t)
$$

where $K_{E S S, i}$ is a coefficient between $p_{i}$ and $e_{i}$. In this paper, it is assumed that the power output of each ESS is rated at 1C, which means $P_{E S S, i}^{\max }$ and $C_{E S S, i}$ have the same value but different 
units, and $\eta_{i}=0.95$ is of the same value for each ESS in one ESA.

Considering the control input dynamics of power state, the ESA can be modelled as a group of homogenous double integral systems as follows:

$$
\left\{\begin{array}{c}
\underset{i}{d}(t)=K_{E S S} p_{i}(t) \\
\operatorname{d}_{t}(t)=u_{i}(t)
\end{array}, \quad i=1,2 \ldots, N .\right.
$$

where $e_{i}$ and $p_{i}$ are the energy and power states and $u_{i}$ is the control input of $i^{\text {th }}$ ESS.

A leader is defined as a controller which can receive the upper level control signal (frequency control signal in this paper). The leader will update the reference power state and energy state of the ESA as follows:

$$
\left\{\begin{array}{c}
\&(t)=K_{E S S} p_{0}(t) \\
p_{0}(t)=\frac{P_{E S A}^{*}(t)}{P_{E S A}^{\max }}
\end{array}\right.
$$

where $e_{0}$ and $p_{0}$ are reference energy and power states of the leader. $P_{E S A}^{*}$ is the system frequency control signal and $P_{E S A}^{\max }$ is the power rating of the ESA, which is equal to $\sum P_{E S S, i}^{\max }$.

Therefore, the research problem can be formulated as designing a leader-follower finite-time consensus control for a group of ESSs with model (19) and (20) in an ESA.

For an ESA formulated by (19) and (20), the leaderfollower finite-time consensus is achieved, if for any initial states, there exists a $T_{0} \in[0, \infty]$, such that

$$
\begin{gathered}
\lim _{t \rightarrow T_{0}}\left\|e_{i}(t)-e_{0}(t)\right\|=0, \lim _{t \rightarrow T_{0}}\left\|p_{i}(t)-p_{0}(t)\right\|=0 \\
e_{i}(t)=e_{0}(t), \quad p_{i}(t)=p_{0}(t), \quad \forall t \geq T_{0}, \quad i=1,2, \ldots N .
\end{gathered}
$$

In order to achieve (21), the leader-follower finite-time consensus control protocol is designed as follows:

$$
\begin{aligned}
& u_{i}(t)=\sum_{j=1}^{N} a_{i j}\left(\operatorname{sig}\left(e_{i}(t)-e_{j}(t)\right)^{\alpha}\right)-g_{i}\left(\operatorname{sig}\left(e_{i}(t)-e_{0}(t)\right)^{\alpha}\right) \\
& -\gamma \sum_{j=1}^{N} a_{i j}\left(\operatorname{sig}\left(p_{i}(t)-p_{j}(t)\right)^{\beta}\right)-g_{i}\left(\operatorname{sig}\left(p_{i}(t)-p_{0}(t)\right)^{\beta}\right)
\end{aligned}
$$

where the function $\operatorname{sig}(x)^{\alpha}=|x|^{\alpha} \operatorname{sign}(x),|x|$ denotes the absolute value of variable $x$, and $\operatorname{sign}($.$) denotes the sign$ function. $\alpha, \beta, \gamma$ are control gains to be selected, $\alpha, \beta, \gamma \in(0,1)$, and $\beta=2 \cdot \alpha /(1+\alpha)$.

The settling time $T_{0}$ satisfies the inequality [27]:

$$
T \leq \frac{V^{1-\alpha}(0)}{c(1-\alpha)}
$$

where $V(x)$ is a positive definite function defined in a neighbourhood of the origin, and real number $c>0$. The stability of system (19) and (20) with the protocol (22) can be proved using Lyapunov's second method by properly selecting Lyapunov function. The detailed proof is given in Appendix.

\section{Numerical Illustrations}

This section aims to show the dynamics of power and energy states and validate control effectiveness under different communication graphs. The control algorithm is tested under two directed communication graphs $\left(\bar{G}_{1}\right.$ and $\left.\overline{\mathcal{G}}_{2}\right)$, as shown in Fig. 3. In $\bar{G}_{1}$, Node 1 (ESS 1 ) is considered as a pinning node which can receive the information from the leader labelled as 0 . Other ESSs are connected in a directed line as shown in Fig. 3 (a). In $\bar{G}_{2}$, Nodes 1 and 8 are both pining nodes While other ESSs are connected in two directed lines as shown in Fig. 3 (b).

The convergence of each ESS with communication graphs $\bar{G}_{1}$ and $\bar{G}_{2}$ are shown in Fig. 4 and Fig. 5, respectively. The figures are drawn with $T_{c a}=0.01 \mathrm{~s}, e_{0}=0.5$ and $\alpha=0.2, \beta=0.33$, $\gamma=0.6$. There is a step change in $p_{0}$ from 0 to 0.5 at $1000 \mathrm{~s}$. The initial energy states of each ESS are [0.46, 0.47, 0.48, $0.49,0.51,0.52,0.53,0.54]$ and initial power state for all is 0 .

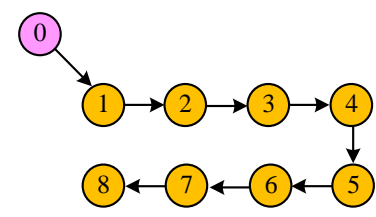

(a) $\overline{G_{1}}$

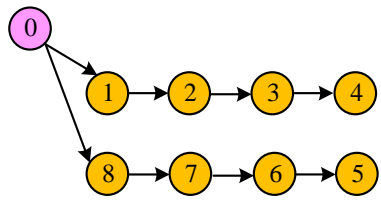

(b) $\bar{G}_{2}$
Fig. 3. Communication network represented by two directed graph.
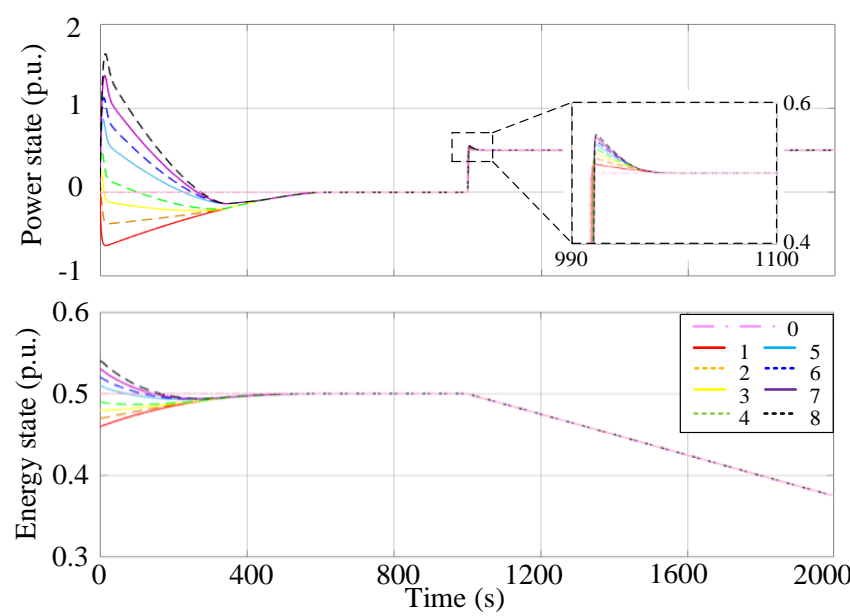

Fig. 4. Convergence speed of ESA with communication graph $\bar{G}_{1}$.
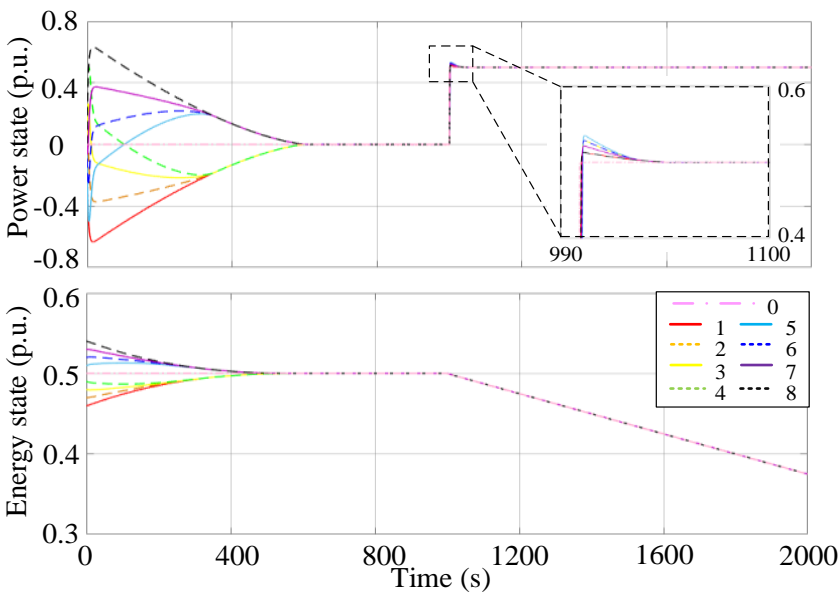

Fig. 5. Converge speed of ESA with communication graph $\bar{G}_{2}$. 
The results in Fig. 4 and Fig. 5 are further illustrated in time sequence:

1) $0<t<1000 \mathrm{~s}$. At the beginning, the energy state of each ESS is not equal. Although the reference power state is 0 during this period, each ESS still need to charge or discharge in order to balance the energy state. As shown in Fig. 4 and Fig. 5, both $\bar{G}_{1}$ and $\bar{G}_{2}$ will converge to consensus and satisfy (21) in finite-time. In Fig. 4, the convergence of ESSs is not symmetrical as graph $\bar{G}_{1}$ is unbalanced. While in Fig. 5, the convergence of ESSs 1-4 and ESSs 5-8 become symmetrical as these two sub-groups have same topologies in graph $\bar{G}_{2}$. The sum of power state for all 8 ESSs in graph $\bar{G}_{2}$ remains 0, which is preferred as it is not desired that the SoC balancing influences the external characteristics of the ESA.

2) $1000 s<t<2000 s$. As the consensus is reached in the first time period, the power and energy states of each ESS are equalized. When there is a step change of $p_{0}$ from 0 to 0.5 at $1000 \mathrm{~s}$, all ESSs start to track the new reference power state. Because the energy states are balanced during this period, the power states of ESSs will converge to $p_{0}$ much faster. The overshoot of $\bar{G}_{2}$ is smaller than $\bar{G}_{1}$, as the connection of last node in $\bar{G}_{2}$ is actually shorter than $\bar{G}_{1}$.

From the numerical illustration, it is validated that both $\bar{G}_{1}$ and $\bar{G}_{2}$ can converge in the finite-time. However, $\bar{G}_{2}$ is preferred as it can reach consensus with smaller overshoot and does not influence the external power response of the ESA. $\bar{G}_{2}$ will be adopted in the simulation tests in Section IV. Generally, the dynamics of the ESA are influenced by factors such as communication rate, graph and control protocol.

\section{Simulation Tests}

In this section, a variety of scenarios are tested to validate the proposed frequency control framework. The LFC model and the proposed ESA are implemented in Matlab/Simulink. To evaluate the performance of the proposed method, both system contingency and normal operation scenarios have been considered. The simulation has been first conducted in single LFC area, and then extended to multi-LFC areas. The LFC system under consideration is a per-unit system with $S_{\text {base }}=80$ MVA. The parameters of the proposed method are shown in Table I. The parameters of the three-area LFC are shown in Table II [19]. The parameters of each test case are shown in Table III. It is assumed that in one control area all the ESSs are aggregated into one ESA. The capacity of the ESA $\left(C_{E S A}\right)$ in Area 1 is $3.2 \mathrm{MWh}$, with eight ESSs of capacity [0.68, 0.6, $0.52,0.44,0.36,0.28,0.2,0.12] \mathrm{MWh}$. While the capacity of the ESA in Area 3 is scaled up to $4.8 \mathrm{MWh}$.

TABLE I

PARAMETERS OF PROPOSED METHOD

\begin{tabular}{c|c}
\hline \hline Parameter & Value \\
\hline Band-pass filter $\left(f_{L}, f_{H}\right)$ & $0.05 \mathrm{~Hz}, 10 \mathrm{~Hz}$ \\
\hline Finite-time consensus control $(\alpha, \beta, \gamma)$ & $0.2,0.33,0.6$ \\
\hline Communication rate $\left(T_{c a}\right)$ & $0.01 \mathrm{~s}$ \\
\hline \hline
\end{tabular}

TABLE II

PARAMETERS OF THREE-AREA POWER SYSTEM

\begin{tabular}{c|c|c|c}
\hline \hline Area No. & 1 & 2 & 3 \\
\hline $2 H$ (p.u./Hz) & 0.1667 & 0.2 & 0.15 \\
\hline$D$ (p.u./Hz) & 0.0015 & 0.002 & 0.001 \\
\hline$T_{a}$ (s) & 5 & 4 & 4.5 \\
\hline$T_{b}$ (s) & 40 & 30 & 40 \\
\hline$T_{g}$ (s) & 0.4 & 0.3 & 0.35 \\
\hline$R$ (Hz/p.u. $)$ & 3 & 3.6 & 2.5 \\
\hline$B$ (p.u./Hz) & 0.8675 & 0.795 & 0.87 \\
\hline$T_{i j}$ (p.u./Hz) & 0.25 & 0.25 & 0.25 \\
\hline$C_{E S A}(\mathrm{MWh})$ & 3.2 & 0 & 4.8 \\
\hline \hline
\end{tabular}

TABLE III

PARAMETERS OF EACH TeSt CASE

\begin{tabular}{|c|c|c|c|}
\hline Test Case & $\begin{array}{c}\text { Load Variation } \\
\left(\Delta P_{L, i}\right) \\
\end{array}$ & $\begin{array}{c}\text { PV Variation } \\
\left(\Delta P_{R E S}\right)\end{array}$ & $\begin{array}{c}\text { ESA } \\
\text { Capacity }\end{array}$ \\
\hline$\# 1$ & At $5 \mathrm{~s}, 0$ to $6.4 \mathrm{MW}$ & N.A. & $\begin{array}{l}\text { 3.2 MWh in } \\
\text { Area } 1\end{array}$ \\
\hline$\# 2$ & Fig. 9 & Fig. 9 & $\begin{array}{c}3.2 \mathrm{MWh} \text { in } \\
\text { Area } 1\end{array}$ \\
\hline$\# 3$ & $\begin{array}{l}\text { At } 5 \mathrm{~s}, 0 \text { to } 6.4 \mathrm{MW} \\
\text { in Area } 1 ; \\
\text { At } 100 \mathrm{~s}, 0 \text { to } 9.6 \\
\text { MW in Area } 3\end{array}$ & N.A. & $\begin{array}{l}\text { 3.2 MWh in } \\
\text { Area 1; } \\
\text { 4.8 MWh in } \\
\text { Area } 3\end{array}$ \\
\hline
\end{tabular}

\section{A. Test Case 1: System Contingency}

In test case 1, the dynamic performance of the proposed method is investigated under system contingency condition in a single area power system. Area 1 with 3.2 MWh ESA, as in Table II, is considered with initial SoCs of ESSs as $50 \%$. A load disturbance of $6.4 \mathrm{MW}$ at $5 \mathrm{~s}$ emulates the contingency.

The system frequency responses under original control scheme without ESA, with ESA but without the disturbance observer, and the proposed control scheme are compared in Fig. 6. Upon occurrence of the disturbance at 5, the frequency of the system begins to drop. The dynamics of the ESA is mainly governed by the proposed disturbance observer other than AGC signal in this condition. As a result, it can be observed that both the frequency nadir and the frequency deviation recovery within the allowable range $( \pm 0.2 \mathrm{~Hz})$ are evidently improved with the proposed control scheme.

The performance of the system disturbance observer is shown in Fig. 7. The parameters of matrixes $\boldsymbol{M}$ and $\boldsymbol{R}$ are chosen as $\boldsymbol{M}=[-1,-1,-1]$ and $\boldsymbol{R}=\operatorname{diag}\{10,10,10\}$ according to (11) and (12). In this condition, the eigenvalues $\lambda_{1}=-22.4$, $\lambda_{2}=-10, \lambda_{3}, 4=-6.4 \pm 4.3 \mathrm{j}$ are assigned to the disturbance observer and the system (11) is stable. It can be noticed that the estimated system disturbance can track the actual system disturbance after a short time. The participation of the ESA in conjunction with the proposed disturbance observer actually reduces the total system disturbance as compared to the load step of 6.4 MW, thereby improving the frequency response.

The power output of each ESS in the ESA during this process is shown in Fig. 8. As shown in Fig. 8, each ESS can respond fast to the load change and reach its maximum power output when there is a large system disturbance. The response speed of each ESS is determined by control protocol (22) as well as communication graphs. The maximum power rating of each ESS is also reflected in Fig. 8, which is the same as the 
capacity of each ESS mentioned before. As the ESA is designed to compensate the power variation rather than the steady-state disturbances, the band-pass filer will reduce the power output of each ESS from 14s. Correspondingly, the disturbance in Fig. 7 will gradually increase after $14 \mathrm{~s}$ due to the reduction of power output from the ESA.

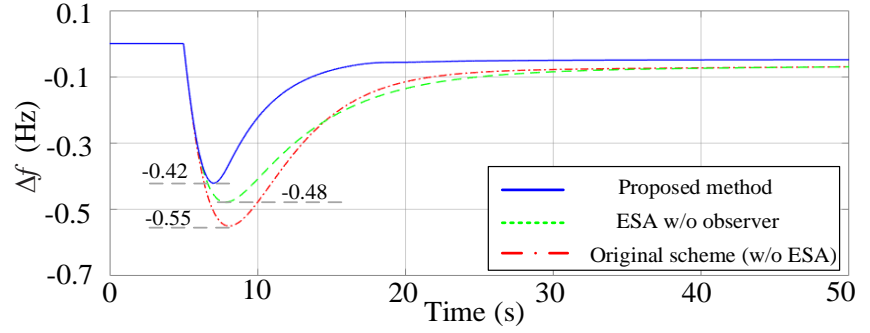

Fig. 6. The frequency response under a step load change.

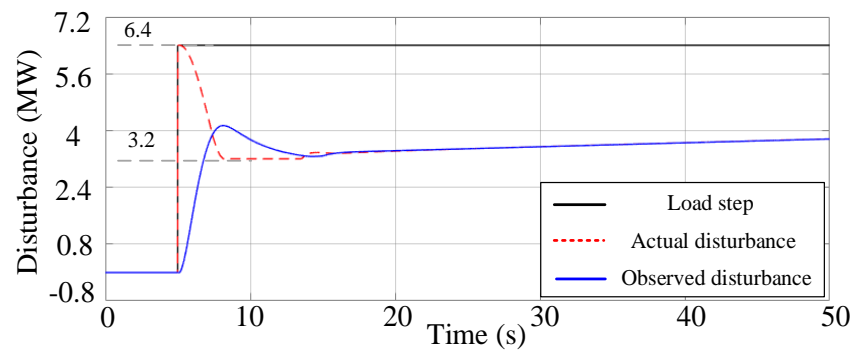

Fig. 7. Perfomance of the proposed disturbance observer.

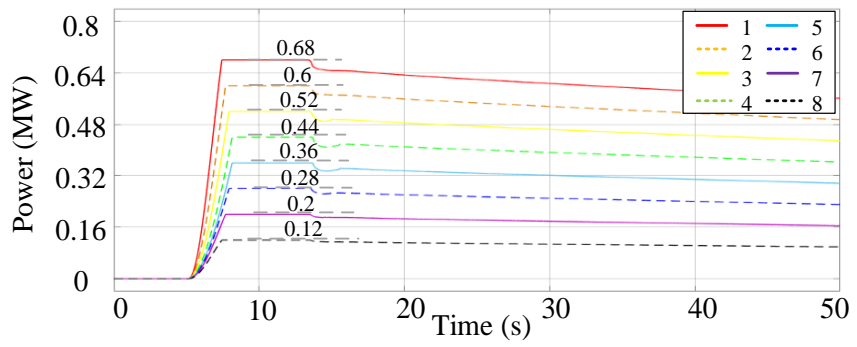

Fig. 8. The power output of each ESS in the ESA.

\section{B. Test Case 2: Normal Operation}

In test case 2, the normal operation study is conducted to further investigate the dynamic performance of the proposed method. The same single area system is considered as in test case 1. The one-hour PV and load variation profiles used in this case are shown in Fig. 9. The PV data with 1-second resolution was measured on June 2012 by EPRI [28]. The PV profile has very high fluctuations while the load profile is much smoother. The initial SoC of each ESS in the ESA is $[46 \%, 47 \%, 48 \%, 49 \%, 51 \%, 52 \%, 53 \%, 54 \%]$. In this test case, the AGC participation factors of ESAs and generators are $\alpha_{\mathrm{ESA}}=0.1$ and $\alpha_{\mathrm{G}}=0.9$, respectively.

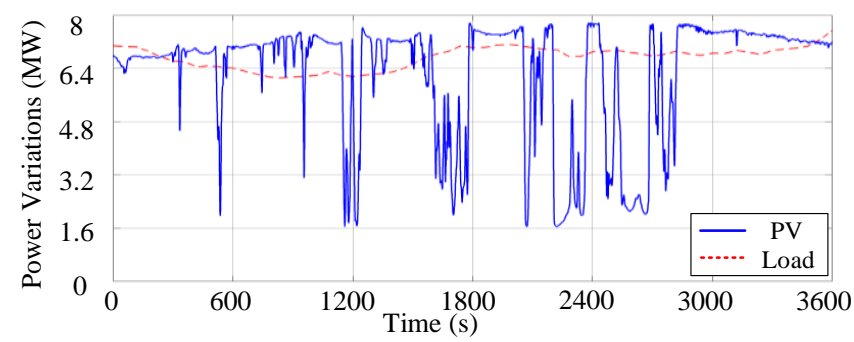

Fig. 9. One-hour PV and load variation profiles.
Fig. 10 (a)-(c) show system frequency deviations with the original control scheme, with ESA but without observer, and with the proposed control scheme, respectively. Comparing Fig. 10 (a)-(c), it can be observed that frequency deviations are mitigated much more effectively with the proposed method. The frequency deviations can be regulated in an allowable range $( \pm 0.2 \mathrm{~Hz})$ with the $3.2 \mathrm{MWh}$ ESA using the proposed method. Furthermore, total power output of the ESA with and without the proposed disturbance observer is compared in Fig. 11. The results are corresponding to (b) and (c) in Fig. 10. The ESA will react faster with the proposed observer, thus the system frequency deviation becomes smaller.

(a) Original scheme (w/o ESA)

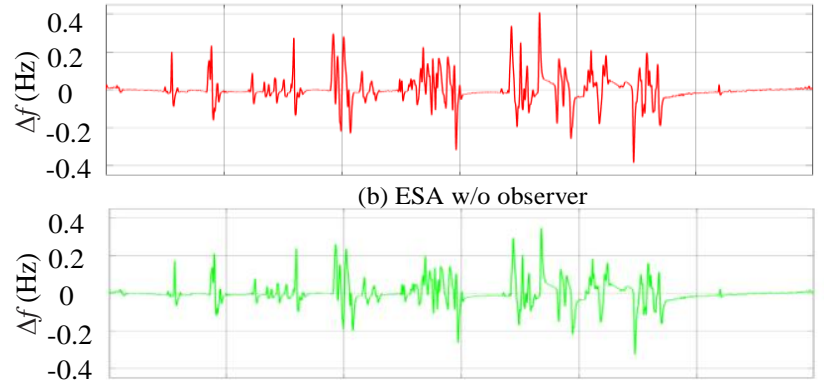

(c) Proposed method (with observer)

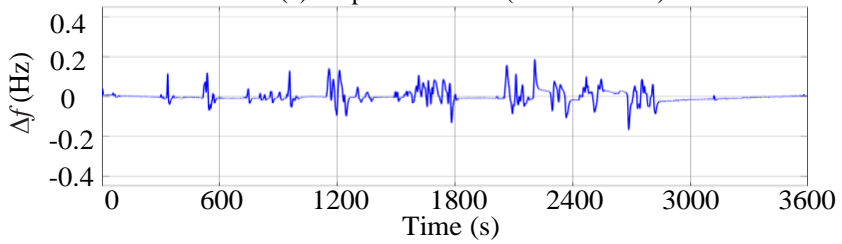

Fig. 10. Frequency deviations with and without proposed method.

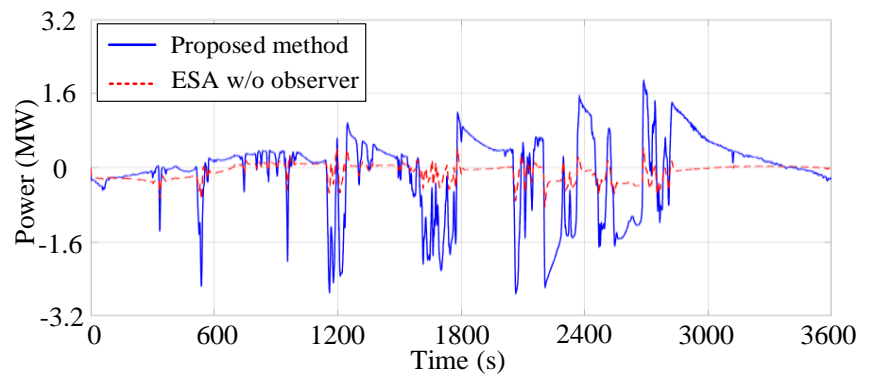

Fig. 11. Total power output of the ESA with and without system disturbance observer.

The power output and SoC profiles of each ESS in the ESA in this test case is shown in Fig. 12 and13, respectively. Initially, the SoC differences between each ESS are large. Therefore, the SoC balancing effect dominates the ESS dynamics. The power sharing among each ESS is much influenced in order to balance SoCs. While the total power output of the ESA still follows the frequency control signal. As the SoC of each ESS gradually become equalized during the operation, the power sharing among each ESS become proportional to their power rating, same as Fig. 8 in test case 1. It can be also observed from Fig. 11 that each ESS in the ESA responds to sudden PV fluctuation to mitigate the frequency deviations. 


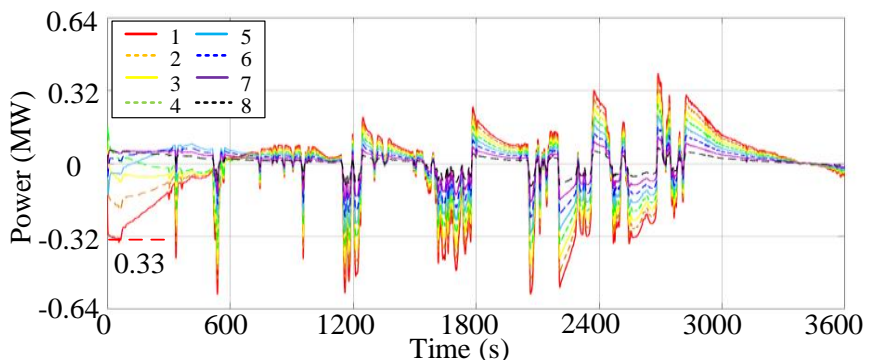

Fig. 12. Power output of each ESS in test case 2

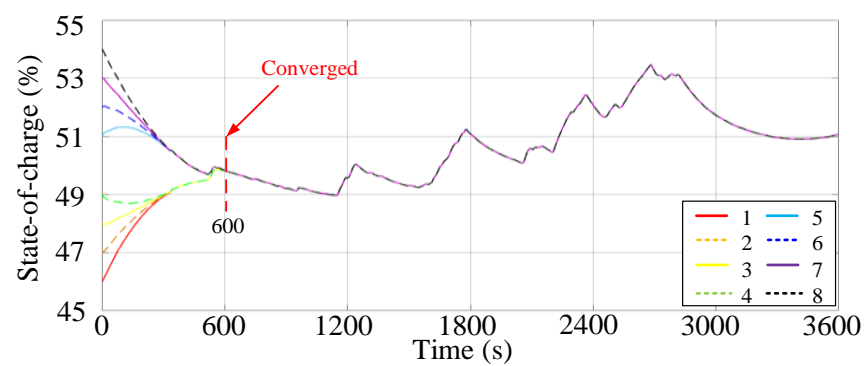

Fig. 13. SoC profile of each ESS in test case 2.

\section{Test Case 3: Multi-Area Power System}

In test case 3, the system contingency study is extended to multi- LFC areas to further investigate the performance of the proposed method. The structure of the studied three-area interconnected power system is depicted in Fig. 14. In test case 3, it is assumed that there is 3.2 MWh ESA in Area 1 and 4.8 MWh ESA in Area 3. There are step load disturbances of 6.4 MW at 5s in Area 1 and 9.6 MW at 100s in Area 3. The proposed disturbance observer will estimate the system distance in each control area for corresponding ESA. Other simulation conditions are the same as in test case 1 .

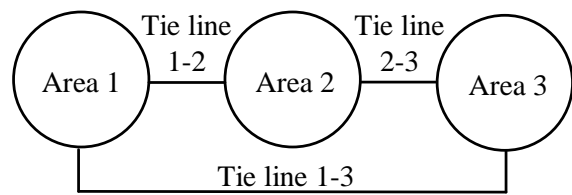

Fig. 14. The structure of the studied three-area power system.

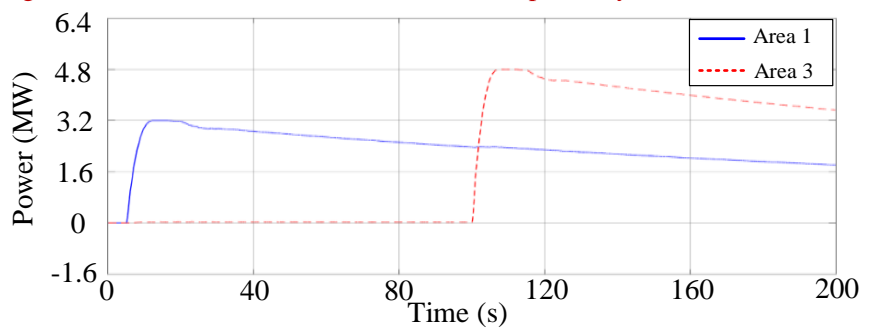

Fig. 15. Total power output of ESAs in Area 1 and Area 3.

Fig. 15 shows the total power output of ESAs under step load changes in Area 1 and Area 3, respectively. The ESAs in Area 1 and Area 3 will respond to the system disturbance in the respective control area, as shown in Fig. 15. Fig. 16 shows the frequency response in each control area of test case 3 . As shown in Fig. 16, the sudden load changes will influence the frequency in not only the same area, but also the interconnected power systems. The frequency response of each control area can be improved with the proposed observer and finite-time consensus-based ESAs. (a) Area 1
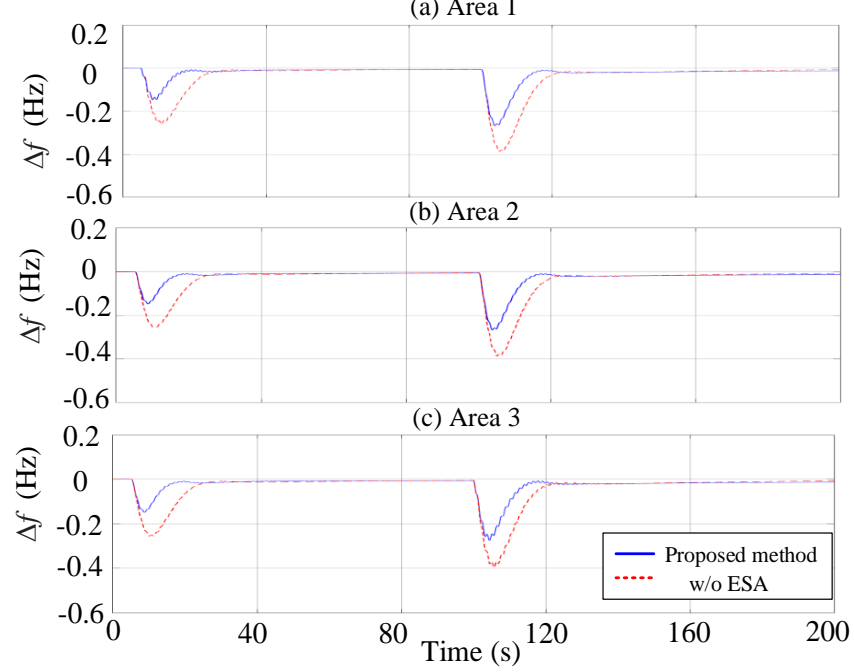

Fig. 16. Frequency response of each control area.

\section{Control Algorithm Comparisons}

In this sub-section, the proposed finite-time consensus control protocol is compared to a commonly utilized consensus control protocol for the second order multi-agent system reported in [29]. Compared to the finite-time approach in this paper, the control protocol in [29] is 'linear' and 'infinite-time'. For system (19) and (20), the control protocol in [29] can be expressed as:

$$
\begin{aligned}
& u_{i}(t)=k_{1} \sum_{j=1}^{N} a_{i j}\left(e_{i}(t)-e_{j}(t)\right)-g_{i}\left(e_{i}(t)-e_{0}(t)\right) \\
& -k_{2} \sum_{j=1}^{N} a_{i j}\left(p_{i}(t)-p_{j}(t)\right)-g_{i}\left(p_{i}(t)-p_{0}(t)\right)
\end{aligned}
$$

where $k_{1}$ and $k_{2}$ are control gains to be selected.

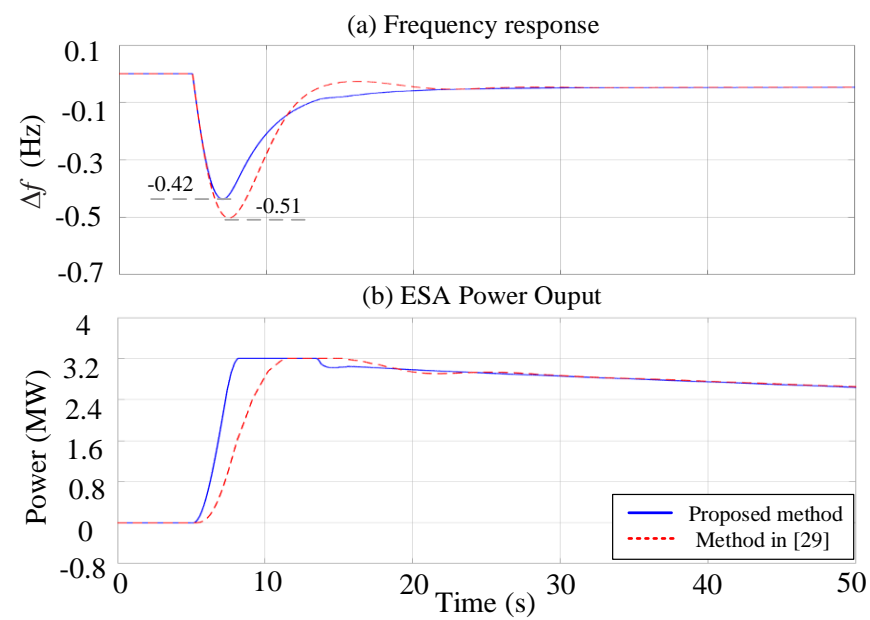

Fig. 17. Comparison with the method in [29]. (a) frequency response. (b) ESA power output.

Firstly, the performance of protocol (24) in [29] and the proposed protocol (22) are compared under system contingency scenario in test case 1 . The comparison results of frequency response and ESA power output are shown in Fig. 17. The control gains of protocol (24) is selected as $k_{1}=35$, $k_{2}=1$. As shown in Fig. 17 (a), it can be found that the proposed method improves the frequency nadir as compared to the method in [29]. Correspondingly, the power output of 
the ESA under the proposed method also reacts faster than the method in [29], as shown in Fig. 17 (b).

Secondly, the protocol (24) in [29] is compared against the proposed control protocol under the normal operation scenario in test case 2. The results of Section IV-B serve as a base reference, i.e., the convergence time for protocol (22) is 600 s (as seen in Fig. 13) and the maximum power overshoot observed is $0.33 \mathrm{MW}$ (for ESS 1 as seen in Fig. 12). The study is undertaken in two steps and the performance in terms of convergence time and system overshoot are compared.

In order to ensure the same convergence time (600s) as in test case 2, the control gains of protocol (24) are tuned as $k_{1}=35, k_{2}=1$. As shown in Fig. 19, the SoC of each ESS can converge at 600s, the same as in Fig. 13. However, the power overshoots in Fig. 18 during the convergence are much higher than in Fig. 12, which even reach the power limits of the ESSs. It can therefore be said that the proposed method presents smaller system overshoot under the same convergence time.

In order to ensure the same maximum power overshoot (0.33 MW of ESS-1) as in test case 2, the control gains of protocol (24) are tuned as $k_{1}=12, k_{2}=1$. As shown in Fig. 20, maximum power overshoot is $0.33 \mathrm{MW}$, the same as in Fig. 12. However, the SoC (or system) in Fig. 21 converges much slower as compared to Fig. 13. Namely, the proposed method can provide faster convergence speed under the same system overshoot.

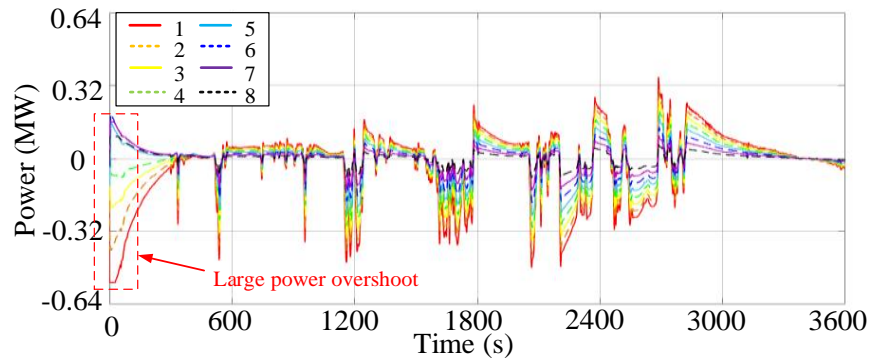

Fig. 18. Power output of each ESS with protocol $(24)\left(k_{1}=35, k_{2}=1\right)$.

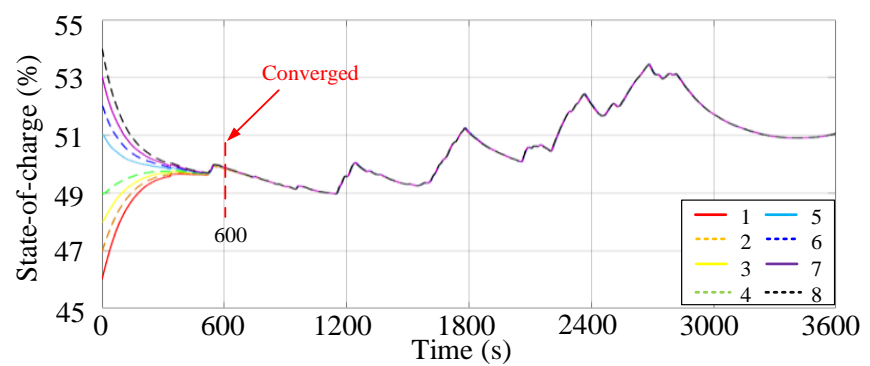

Fig. 19. SoC profile of each ESS with protocol (24) $\left(k_{1}=35, k_{2}=1\right)$.

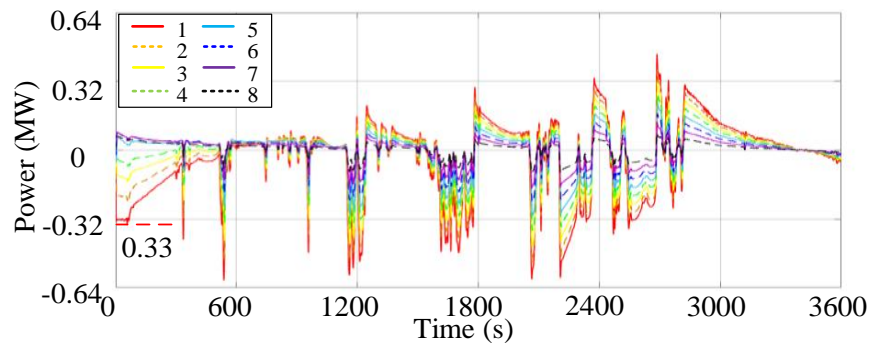

Fig. 20. Power output of each ESS with protocol $(24)\left(k_{1}=12, k_{2}=1\right)$.

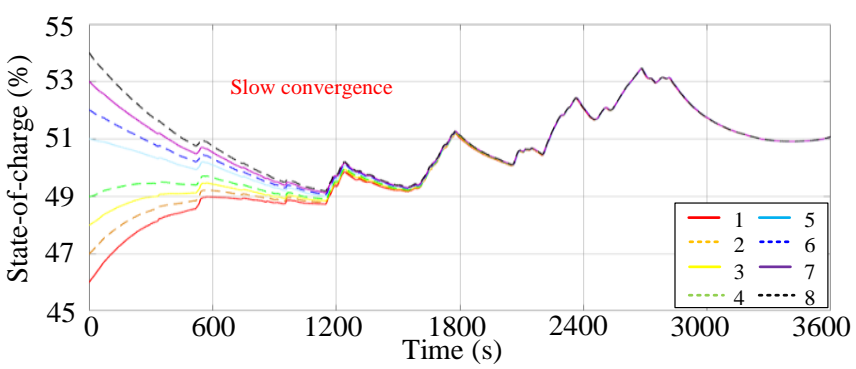

Fig. 21. SoC profile of each ESS with protocol (24) $\left(k_{1}=12, k_{2}=1\right)$.

The advantages of the finite-time approach compared with the common used method in [29] are summarized as follow. In case of a system contingency, the ESA with the proposed finite-time approach has faster response of power output, thus far improves the system frequency response. Under the normal operation, the ESS with proposed finite-time approach presents smaller power overshoot and faster convergence. Smaller power overshoot and faster convergence are desired for operation of ESA as: (i) ESSs within the ESA have a lower chance to reach the power limits during the operation and (ii) any newly joined ESS can attain faster SoC balancing, ensuring plug-and-play capability.

\section{CONCLUSION}

In this paper, a new frequency control framework with finite-time consensus-based ESA has been proposed to improve the power system frequency response in the presence of stochastic renewable power generation. The total frequency control signal for ESA is determined by both AGC commands and the proposed disturbance observer. The control protocol using a leader-follower finite-time consensus algorithm has been proposed to aggregate ESSs in one control area into an ESA. The dynamic response or external characteristic of the ESA is determined by its communication graph and control protocol settings.

Simulation results have shown that the system frequency response can be improved by using the proposed disturbance observer and finite-time consensus-based ESA. The proposed method is effective for both power system contingency and normal operation conditions. The ESA can track the frequency control signal and the SoC of each ESS can be balanced in finite-time during the operation. The proposed method has been extended to multi-area power systems. The comparative studies validate the advantages and necessity of the proposed method. The research results validate that the proposed method is an effective way to incorporate widespread ESSs for power system frequency control.

\section{APPENDIX}

\section{A. Globally Asymptotically Stability}

Let $\hat{p}_{i}(t)=p_{i}(t)-p_{0}(t), \hat{e}_{i}(t)=e_{i}(t)-e_{0}(t)$, the system (19) and (20) becomes

$$
\left\{\begin{array}{c}
e_{i}^{\&}(t)=K_{E S S} \hat{p}_{i}(t) \\
\hat{f}_{i}^{\&}(t)=\hat{u}_{i}(t)
\end{array}, \quad i=1,2 \ldots, N .\right.
$$


The control protocol (22) becomes

$$
\begin{aligned}
& \hat{u}_{i}(t)=\sum_{j=1}^{N} a_{i j}\left[\left(\operatorname{sig}\left(\hat{e}_{i}(t)-\hat{e}_{j}(t)\right)^{\alpha}\right)-\gamma\left(\operatorname{sig}\left(\hat{p}_{i}(t)-\hat{p}_{j}(t)\right)^{\beta}\right)\right] \\
& -g_{i}\left[\left(\operatorname{sig}\left(\hat{e}_{i}(t)\right)^{\alpha}-\gamma\left(\operatorname{sig}\left(\hat{p}_{i}(t)\right)^{\beta}\right)\right)\right]
\end{aligned}
$$

Choose a Lyapunov function $V=V_{1}+V_{2}+V_{3}$

$$
\begin{aligned}
& V_{1}=\frac{1}{2} \sum_{i=1}^{n} \hat{p}_{i}^{2}, \\
& V_{2}=\sum_{i=1}^{n} \sum_{j=1}^{n} \int_{0}^{\hat{e}_{i}-\hat{e}_{j}} a_{i j}\left(\operatorname{sig}(s)^{\alpha}\right) d s, \\
& V_{3}=\sum_{i=1}^{n} \int_{0}^{\hat{e}_{i}} \hat{p}_{i}\left(\operatorname{sig}(s)^{\alpha}\right) d s .
\end{aligned}
$$

Take the derivative of $V$

$$
\begin{aligned}
& \qquad \&=V_{1}^{\&}+V_{2}^{\&}+V_{3}^{\&} \\
& =\sum_{i=1}^{N} \hat{p}_{i}\left[\sum _ { j = 1 } ^ { N } a _ { i j } \left(\operatorname{sig}\left(\hat{e}_{i}-\hat{e}_{j}\right)^{\alpha}-\gamma\left(\operatorname{sig}\left(\hat{p}_{i}-\hat{p}_{j}\right)^{\beta}\right)\right.\right. \\
& \left.-g_{i}\left(\operatorname{sig}\left(\hat{e}_{i}\right)^{\alpha}-\gamma \operatorname{sig}\left(\hat{p}_{i}\right)^{\beta}\right)\right] \\
& \left.+\frac{1}{2} \sum_{i=1}^{N} \sum_{j=1}^{N} a_{i j}\left(\hat{p}_{i}-\hat{p}_{j}\right) \operatorname{sig}\left(\hat{e}_{i}-\hat{e}_{j}\right)^{\alpha}\right) \\
& +\sum_{i=1}^{N} g_{i} \hat{p}_{i} \operatorname{sig}\left(\hat{e}_{i}\right)^{\alpha} \\
& =\sum_{i=1}^{N} \hat{p}_{i} \gamma\left[\sum_{j=1}^{N} a_{i j} \operatorname{sig}\left(\hat{p}_{i}-\hat{p}_{j}\right)^{\beta}-g_{i} \operatorname{sig}\left(\hat{p}_{i}\right)^{\beta}\right] \\
& \left.=\frac{1}{2} \sum_{i=1}^{N} \sum_{j=1}^{N} a_{i j}\left(\hat{p}_{i}-\hat{p}_{j}\right) \operatorname{sig}\left(\hat{p}_{i}-\hat{p}_{j}\right)^{\beta}\right) \\
& -\sum_{i=1}^{n} g_{i} \hat{p}_{i} \operatorname{sig}\left(\hat{p}_{i}\right)^{\beta} \\
& \left.\leq \frac{1}{2} \sum_{i=1}^{N} \sum_{j=1}^{N} a_{i j}\left(\hat{p}_{i}-\hat{p}_{j}\right) \operatorname{sig}\left(\hat{p}_{i}-\hat{p}_{j}\right)^{\beta}\right) \\
& \leq 0
\end{aligned}
$$

According to Lyapunov's second method for stability, $V \geq 0, \quad \quad \& \leq 0$. Therefore, the system (25) with the control protocol (26) is stable.

Furthermore, note that $L^{\&}=0$, if and only if $\hat{p}_{i}=\hat{p}_{j}=0$, which implies $f_{i}^{\&}=0, \forall i, j=1,2 \ldots N$.

$$
\begin{aligned}
& \hat{p}_{i}^{\&}(t)=\sum_{j=1}^{N} a_{i j}\left(\operatorname{sig}\left(\hat{e}_{i}(t)-\hat{e}_{j}(t)\right)^{\alpha}-\gamma \operatorname{sig}\left(\hat{p}_{i}(t)-\hat{p}_{j}(t)\right)^{\beta}\right) \\
& \left.-g_{i}\left(\operatorname{sig}\left(\hat{e}_{i}(t)\right)^{\alpha}-\gamma \operatorname{sig}\left(\hat{p}_{i}(t)\right)^{\beta}\right)\right] \\
& =\sum_{j=1}^{N} a_{i j} \operatorname{sig}\left(\hat{e}_{i}(t)-\hat{e}_{j}(t)\right)^{\alpha}-g_{i} \operatorname{sig}\left(\hat{e}_{i}(t)\right)^{\alpha} \\
& =0
\end{aligned}
$$

It follows

$$
\begin{aligned}
& \sum_{i=1}^{N} \hat{p}_{i}\left[\sum_{j=1}^{N} a_{i j} \operatorname{sig}\left(\hat{e}_{i}(t)-\hat{e}_{j}(t)\right)^{\alpha}-g_{i} \operatorname{sig}\left(\hat{e}_{i}(t)\right)^{\alpha}\right] \\
& =-\frac{1}{2} \sum_{i=1}^{N} \sum_{j=1}^{N} a_{i j}\left(\hat{e}_{i}-\hat{e}_{j}\right) \operatorname{sig}\left(\hat{e}_{i}-\hat{e}_{j}\right)^{\alpha}-\sum_{i=1}^{N} g_{i} \hat{e}_{i} \operatorname{sig}\left(\hat{e}_{i}\right)^{\alpha} \\
& =0
\end{aligned}
$$

Equality above implies that $\hat{e}_{i}=\hat{e}_{j}=0$ which means the system will converge to $e_{i} \rightarrow e_{0}, p_{i} \rightarrow p_{0}$ at steady-state. Thus the system (25) with control protocol (26) is globally asymptotically stable.

\section{B. Locally Finite-time Stability}

If the equilibrium of a control is globally asymptotically stable and locally finite-time convergent, then the control is globally finite-time stable [30]. This follows the principle that globally asymptotical stability implies finite-time convergence to any given bounded neighborhood of the equilibrium.

With the previous proof of globally asymptotic stability, the finite-time convergence of the system (25) with control protocol (26) is proved as below.

Lemma 1. (Lasalle's Invariance Principle)

Let $x(t)$ be a solution of $f(x), x(0)=x_{0} \in R^{k}$, where $f: U \rightarrow R^{k}$ is continuous with $U$ an open subset of $R^{k}$, and let $V: U \rightarrow R^{k}$ be a locally Lipschitz function such that $D^{+} V(x(t))$ $\leq 0$, where $D^{+}$denotes the upper Dini derivative. Then, denoting the positive limit set as $\Lambda^{+}\left(x_{0}\right), \Lambda^{+}\left(x_{0}\right) \cap U$ is contained in the union of all solutions that remain in $S=\{x \in$ $U: D^{+} V(x)=0$ \}.

Definition 1. (Homogeneity with dilation)

A function $V(x)$ of $x \in R^{k}$ is homogeneous of degree homogeneous of degree $\sigma \geq 0$ with dilation coefficients $\left(r_{1}, \ldots\right.$, $\left.r_{k}\right)$, if $V\left(\varepsilon^{r_{1}} x_{1}, \ldots, \varepsilon^{r_{k}} x_{k}\right)=\varepsilon^{\sigma} V(x), \varepsilon>0$. If $r_{1}=\ldots=r_{k}=1$, then the dilation is called trivial.

Consider $k$-dimensional system

$$
\&=f(x), x=\left(x_{1}, \ldots, x_{k}\right)^{T} \in R^{k}
$$

A continuous vector field $f(x)=\left(f_{1}(x), \ldots, f_{k}(x)\right)^{\mathrm{T}}$ is homogeneous of degree $\sigma \in R$ with dilation $\left(r_{l}, \ldots, r_{k}\right)$, if $f_{i}\left(\varepsilon^{r_{1}} x_{1}, \ldots, \varepsilon^{r_{k}} x_{k}\right)=\varepsilon^{\sigma} f_{i}(x), \varepsilon>0$.

System (27) is called homogeneous if its vector field is homogeneous. Moreover,

$$
\mathcal{H}=f(x)+\hat{f}(x), \hat{f}(0)=0, x \in R^{k}
$$

is called locally homogeneous if $\mathrm{f}$ is homogeneous of degree $\sigma$ $\in R$ with dilation $\left(r_{l}, \ldots, r_{k}\right)$ and $\hat{f}$ is a continuous vector field satisfying

$$
\lim _{\varepsilon \rightarrow 0} \frac{\hat{f}_{i}\left(\varepsilon^{r_{1}} x_{1}, \ldots, \varepsilon^{r_{k}} x_{k}\right)}{\varepsilon^{\sigma+r_{i}}}=0, \forall x \neq 0
$$

Lemma 2. Suppose system (27) is homogeneous of degree $\sigma$ with dilation $\left(r_{1}, \ldots, r_{k}\right), f$ is continuous and $x=0$ is its asymptotically stable equilibrium. If homogeneity degree $\sigma<$ 0 , the equilibrium of system (27) is finite-time stable. 
Moreover, if (29) holds, then the equilibrium of system (28) is locally finite-time stable.

The we can get system with variables $\left(\hat{e}_{1}, \hat{e}_{2}, \ldots, \hat{e}_{n}, \hat{p}_{1}, \hat{p}_{2}, \ldots, \hat{p}_{n}\right)$ is homogeneous of degree $\kappa=\alpha-1<0 \quad$ with dilation $(2,2, \ldots, 2,1+\alpha, 1+\alpha, \ldots, 1+\alpha)$. Therefore, according to Lemma 2 , the system with control law is locally finite-time stable.

Therefore, system (25) with control protocol (26) is globally finite-time stable. In other words, we have $\hat{e}_{i}-\hat{e}_{j} \rightarrow 0, \hat{p}_{i}-\hat{p}_{j} \rightarrow 0, \forall i, j=1, \ldots, N$ in finite-time. This completes the proof.

\section{REFERENCES}

[1] A. M. Bouzid, et. al., "A survey on control of electric power distributed generation systems for microgrid applications", Renewable Sustainable Energy Reviews, vol. 44, pp. 751-766, Apr. 2015.

[2] Next-generation electricity: The emerging negawatt and micropower revolutions. [Online]. Available: http://sg.sg/N80b2m

[3] H. Bevrani, A. Ghosh, and G. Ledwich, "Renewable energy sources and frequency regulation: Survey and new perspectives," IET Renewable Power Generation, vol. 4, no. 5, pp. 438-457, Sep. 2010.

[4] V. Knap, et. al., "Sizing of an energy storage system for grid inertial response and primary frequency reserve", IEEE Trans. Power systems, vol. 31, No. 5, pp. 3447-3456, Dec. 2015.

[5] P. Kundur, Power System Stability and Control. New York, NY, USA: McGraw-Hill, 1994.

[6] S. Vazquez, S. M. Lukic, E. Galvan, L. G. Franquelo, and J. M. Carrasco, "Energy storage systems for transport and grid applications," IEEE Trans. Industrial Electronics, vol. 57, no. 12, pp. 3881-3895, Dec. 2010.

[7] Launch of Singapore's First Utility-Scale Energy Storage System. [Online]. Available: https://www.ema.gov.sg/cmsmedia/Energy\%20 Storage\%20Systems\%20Test-Bed\%20Media\%20Release.pdf

[8] X. J. Li, D. Hui, and X. K. Lai, "Battery energy storage station (BESS)based smoothing control of photovoltaic (PV) and wind power generation fluctuations," IEEE Trans. Sustainable Energy, vol. 4, no. 2, pp. 464-473, Mar. 2013.

[9] L. Miao, J. Y. Wen, C. Y. Yue, et al., "Coordinated control strategy of wind turbine generator and energy storage equipment for frequency support," IEEE Trans. Industry Applications, vol. 51, no. 4, pp. 27322742, Jan. 2013.

[10] U. C. Chukwu and S. M. Mahajan, "V2G parking lot with PV rooftop for capacity enhancement of a distribution system," IEEE Trans. Sustainable Energy, vol. 5, no. 1, pp. 119-127, Sep. 2013.

[11] X. H. Liu, A. Aichhorn, L. M. Liu, and H. Liu, "Coordinated control of distributed energy storage system with tap changer transformers for voltage rise mitigation under high photovoltaic penetration," IEEE Trans. Smart Grid, vol. 3, no. 2, pp. 897-906, Feb. 2012.

[12] M. J. E. Alam, K. M. Muttaqi, and D. Sutanto, "Mitigation of rooftop solar PV impacts and evening peak support by managing available capacity of distributed energy storage systems," IEEE Trans. Power Systems, vol. 28, no. 4, pp. 3874-3884, Nov. 2013.

[13] L. Gkatzikis, K. Iordanis, and S. Theodoros, "The role of aggregators in smart grid demand response markets" IEEE Journal on Selected Areas in Communications, no. 31, vol .7, pp. 1247-1257, Jul. 2013.

[14] S. Burger, et al. "The value of aggregators in electricity systems," MIT working paper, 2016.

[15] S. Q. Zhang, Y. Mishra, and M. Shahidehpour, "Fuzzy-logic based frequency controller for wind farms augmented with energy storage systems," IEEE Trans. Power Systems, vol. 31, no .2, pp. 1595-1603, Mar. 2016

[16] S. J. Lee, et al. "Coordinated control algorithm for distributed battery energy storage systems for mitigating voltage and frequency deviations," IEEE Trans. on Smart Grid, vol. 7, no. 3, pp. 1713-1722, May 2016

[17] J. Zhong, et al. "Coordinated control for large-scale EV charging facilities and energy storage devices participating in frequency regulation," Applied Energy, vol. 123, pp. 253-262, Jun. 2014.
[18] H. H. Xin, Z. H. Qu, J. Seuss, and A. Maknouninejad "A self-organizing strategy for power flow control of photovoltaic generators in a distribution network," IEEE Trans. Power Systems, vol. 26, no. 3, pp. 1462-1473, Aug. 2011.

[19] G. Mokhtari, A. Ghosh, G. Nourbakhsh, and G. Ledwich, "Smart robust resources control in LV network to deal with voltage rise issue," IEEE Trans. Sustainable Energy, vol. 4, no. 4, pp. 1043-1050, Oct. 2013.

[20] J. Q. Hu, et al. "Improving frequency stability based on distributed control of multiple load aggregators," IEEE Trans. Smart Grid, Nov. 2015.

[21] Wen, Guanghui, et al. "Frequency regulation of source-grid-load systems: A compound control strategy," IEEE Trans. Industrial Informatics, vol. 12, no.1, pp. 69-78, Feb. 2016.

[22] F. Guo, C. Wen, J. Mao, and Y. Song, "Distributed secondary voltage and frequency restoration control of droop-controlled inverter-based microgrids," IEEE Trans. Industrial Electronics, vol. 62, no.7, pp. 43554364, 2015.

[23] S. Zuo, A. Davoudi, Y. Song, and F. L. Lewis, "Distributed finite-time voltage and frequency restoration in islanded AC microgrids" IEEE Trans. Industrial Electronics, vol. 63, no. 10, pp. 5988-5997, 2016.

[24] D. Ginoya, P. D. Shendge, and S. B. Phadke, "State and extended disturbance observer for sliding mode control of mismatched uncertain system," Journal of Dynamic Systems, Measurement, and Control, vol. 137, no. 7, Jul. 2015.

[25] C. Luo, H. Banakar, B. Shen and B. T. Ooi, "Strategies to Smooth Wind Power Fluctuations of Wind Turbine Generator," in IEEE Trans. Energy Conversion, vol. 22, no. 2, pp. 341-349, June 2007.

[26] H. Zhang, F. L. Lewis, and Z. Qu, "Lyapunov, adaptive, and optimal design techniques for cooperative systems on directed communication graphs," IEEE Trans. Industrial Electronics, vol. 59, no. 7, pp. 30263041, 2012.

[27] S. Yu, and X. Long, "Finite-time consensus for second-order multi-agent systems with disturbances by integral sliding mode," Journal of Automatica, vol. 54, pp. 158-165, Apr. 2015.

[28] 1-second resolution data, EPRI. [Online]. Available: http://dpv.epri.com/measurement_data.html.

[29] S. Qiang, J. Cao, and W. Yu, "Second-order leader-following consensus of nonlinear multi-agent systems via pinning control," Systems \& Control Letters, vol. 59, no.9, pp. 553-562, Sep. 2010.

[30] X. Wang, and Y. Hong, "Finite-time consensus for multi-agent networks with second-order agent dynamics," IFAC Proceedings Volumes, vol. 41, no .2, pp. 15185-15190, Dec. 2018.

Yu Wang (S'12-M'16) received the B.Eng. degree from Wuhan University, Wuhan China in 2011, and the M.Sc. and Ph.D. degree from Nanyang Technological University, Singapore in 2012 and 2016, respectively. He is currently a research fellow in Nanyang Technological University, Singapore. His research interests include distributed control in power systems, energy storage systems and smart grids.

Yan Xu (S'10-M'13) received the B.E. and M.E degrees from South China University of Technology, Guangzhou, China in 2008 and 2011, respectively, and the Ph.D. degree from The University of Newcastle, Australia, in 2013. $\mathrm{He}$ is now the Nanyang Assistant Professor with the School of Electrical and Electronic Engineering, Nanyang Technological University, Singapore. He was previously with the University of Sydney, Australia. His research interests include power system stability and control, microgrid and multienergy network, and data-analytics for smart grid.

Yi Tang (S'10-M'14) received the B.Eng. degree in electrical engineering from Wuhan University, Wuhan, China, in 2007, and the M.Sc. and Ph.D. degrees in power engineering from the School of Electrical and Electronic Engineering, Nanyang Technological University, Singapore, in 2008 and 2011, respectively.

Kai Liao (M'16) received the B.E. and Ph.D. degrees from Southwest Jiaotong University, Chengdu, China, in 2011 and 2016, respectively. He is currently a Research Fellow in the School of Electrical and Electronic Engineering, Nanyang Technological University, Singapore. His research interests include power system stability and control, and renewable energy.

Mazheruddin H. Syed (S'11) received his B.E. degree in Electrical and Electronics Engineering from Osmania University, India, in 2011 and his M.Sc. degree in Electrical Power Engineering from Masdar Institute of Science and Technology, UAE. Presently, he is a research assistant at the 
Institute for Energy and Environment, the University of Strathclyde, Scotland, working towards his Ph.D. His research interests include demand side management, decentralized and distributed control, and power hardware in the loop simulations.

Efren Guillo-Sansano (S'13) received his M.Sc. degree in Electrical Power Engineering from the University of Strathclyde, Glasgow, Scotland, in 2013.

$\mathrm{He}$ is currently a research assistant with the Institute for Energy and Environment at the University of Strathclyde, Scotland, where he is pursuing a Ph.D. degree in electrical power systems. His current research interests include the testing and validation of distributed energy resources and power electronics, power hardware in the loop, distributed control, and ancillary services.

Graeme M. Burt (M'95) received the B.Eng. degree in electrical and electronic engineering, and the Ph.D. degree in fault diagnostics in power system networks from the University of Strathclyde, Glasgow, U.K., in 1988 and 1992, respectively. He is currently a Professor of electrical power systems at the University of Strathclyde where he co-directs the Institute for Energy and Environment, directs the Rolls-Royce University Technology Centre in Electrical Power Systems, and is lead academic for the Power Networks Demonstration Centre (PNDC). In addition, he serves as spokesperson for the board of DERlab e.V., the association of distributed energy laboratories. His research interests include the areas of power system protection and control, distributed energy, and experimental validation. 